\title{
Real-time Intraday Traffic Volume Forecasting - A Hybrid Application Using Singular Spectrum Analysis and Artificial Neural Networks
}

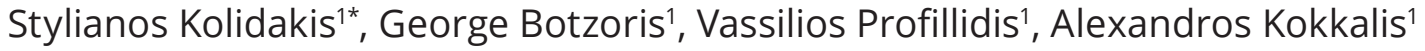 \\ ${ }^{1}$ Section of Transportation Engineering, Department of Civil Engineering, Faculty of Engineering, \\ Democritus University of Thrace, Kimmeria Campus - Building B, 67100 Xanthi, Greece \\ *Corresponding author, e-mail: skolidak@ee.duth.gr
}

Received: 01 April 2019, Accepted: 04 June 2019, Published online: 11 September 2019

\begin{abstract}
The present paper provides a comparative evaluation of hybrid Singular Spectrum Analysis (SSA) and Artificial Neural Networks (ANN) against conventional ANN, applied on real time intraday traffic volume forecasting. The main research objective was to assess the applicability and functionality of intraday traffic volume forecasting, based on toll station measurements. The proposed methodology was implemented and evaluated upon a custom developed forecasting software toolbox, based on the software Mathworks MatLab, by using real data from lasmos-Greece toll station. Experimental results demonstrated a superior ex post forecasting accuracy of the proposed hybrid forecasting methodology against conventional ANN, when compared to performance of usual statistical criteria (Mean Absolute Error, Mean Squared Error, Root Mean Squared Error, Coefficient of Determination R², Theil's inequality coefficient). The obtained results revealed that the hybrid model could advance forecasting accuracy of a conventional ANN model in intraday traffic volume forecasting, while embedding hybrid forecasting algorithm in an Intelligent Transport System could provide an advanced decision support module for transportation system maintenance, operation and management.
\end{abstract}

Keywords

singular spectrum analysis, artificial neural network, traffic analysis, ex post forecast, transportation

\section{Introduction}

\subsection{Rationale}

The importance of analyzing and forecasting transportation demand in order to be able to predict the expected number of trips in a given road network must not be overlooked. Transportation demand forms the essential input in any decision related to the creation, rehabilitation and management of road infrastructure and traffic facilities.

One of the most popular quantitative traffic volume forecasting methods is time series analysis, which mainly uses data from the past to evaluate the future response of traffic volume, without analysis of any other parameter that affect the traffic volume. This means that research focuses on the transformation and decomposition of time series in order to excerpt useful information for the future exploitation, taking into consideration the fact that, besides time, all other factors that affect traffic volume will remain the same in the future (Box et al., 2015).

In recent years, a wide range of methodologies has been implemented for traffic volume forecasting, depending basically upon the type of the available data (Pamuła 2012; Teodorović and Janić, 2017; Vlahogianni et al., 2014). An example of short-term traffic volume forecasting can be the forecasting for the next days, given a time series of the previous days traffic volume. In the present approach, historical data are collected and analyzed with the application of a forecasting model, based on hybrid Singular Spectrum Analysis (SSA) and Artificial Neural Networks (ANN) methods (hybrid SSA-ANN). Then, this model is extrapolated for the forecast of future traffic demand (Zhang and Qi, 2005).

\subsection{Research objectives}

Computational intelligence, engaging hybrid SSA-ANN is an effective approach for modeling complex forecasting phenomena (Andersson et al., 2017; Profillidis and Botzoris, 2018). Hybrid methodology provides a robust potential for modeling, analyzing and forecasting, compared to conventional ANN models (Profillidis 
and Botzoris, 2018; Vlahogianni et al., 2008). The main research goals are to provide empirical evidence for the effectiveness of hybrid SSA-ANN modeling and forecasting real time intraday traffic volume. The present paper examines the following research questions:

1. Is the hybrid SSA-ANN methodology able to model and achieve competent forecasting performance for intraday traffic volume?

2. Is the hybrid SSA-ANN forecasting approach an efficient methodology that provides better forecasting ability than conventional ANN forecasting models?

3. Can this methodology be incorporated as an interactive module, enabling decision support actions in an Intelligent Transport System (ITS) for one-step ahead real time forecasting?

\section{Literature review}

Blinova (2007) used ANN to analyze the possibility to forecast the expansion of intraregional and interregional passenger air traffic flow in Russia. The authors presented a literature review for time-lagged $\mathrm{ANN}$, focusing on standard Back Propagation (BP) learning algorithm, and created matrices of origin-destination passenger flows for all Russian airports. The experimental results provided satisfactory forecasts of passenger traffic demand.

Hassani (2007) applied SSA techniques in monthly accidental deaths time series in the United States and compared them with traditional forecasting methodologies; this proved to be an accurate approach. SSA was applied to the time series of accidental deaths for the period 1973-1978, and the results indicated a superior forecasting method in terms of Mean Absolute Error (MAE) and Mean Relative Absolute Error (MRAE) (for the various evaluation criteria, see $\S 4.2$ ).

Aleksandrov (2009) provided an easy to use methodology for trend extraction by using SSA, which permitted trend extraction when oscillations and noise do occur, and it had only two significant parameters; the scale of extracted trend and the threshold value. The author performed trend extraction by introducing time series spectral analysis and then presented the low-frequency boundary selection and threshold definition. Simulation examples were executed by using data related to unemployment time series in Alaska, and proved the easiness in use and accuracy in forecasting of the proposed methodology.

Hassani and Thomakos (2010) examined the theoretical and methodological approach of SSA forecasting economic-financial time series. The authors proposed two new SSA versions and provided a plethora of simulation results and applications, combined with other forecasting methodologies. The research provided an extensive review of SSA in the area of economic-financial time series. Both the theoretical developments and the results presented a powerful support for SSA potentials in forecasting economic-financial time series.

Pamuła (2012) presented a traffic flow intensity analysis for traffic flow forecast, by employing ANN. ANN was able to recognize and classify flow classes into four major categories. The proposed ANN was adequate for traffic flow classification, since it recognized correctly traffic flow categories. The author suggested that this research indicated ANN forecasting capability to be valuable for decision support on traffic control or management strategy in ITS.

Pamuła (2013) presented a forecasting method for shortterm traffic volume applications. The research scope was to present in detail an accurate method which could be incorporated as a module in ITS. All ANN models were evaluated for three traffic volume classes (working days, Saturdays and Sundays), but the finest outcomes were acquired for working days data. Evaluation was performed by using statistical criteria such as the Root Mean Squared Error (RMSE), MAE, and the Mean Absolute Percentage Error (MAPE). The best forecasting model was identified and was significant for urban traffic planning.

Wu et al. (2013) compared Autoregressive Integrated Moving Average (ARIMA) models with Generalized Regression Neural Networks (GRNN) in order to solve traffic forecasting problems and alleviate massive traffic transportation network problems. Both approaches were analyzed, and the paper focused on extracting daily traffic characteristics from initial time series. Experimental results suggest that ARIMA performed better than GRNN, in terms of forecasting ability.

Schimbinschi et al. (2015) used big data and machine learning techniques to forecast the traffic volume in the city of Melbourne for a period of six years. Measurements were aggregated in 15 minutes intervals, for the same road direction for 96 different sensors. Models were built using both spatial and temporal considerations such as data from past week, day of the week and traffic from nearby sensors. The experimental results highlighted the necessity to separate initial time series into working and non-working days subsets in order to increase the forecasting accuracy. It seemed that spatial characteristics influence more than temporal characteristics the forecasting performance of the proposed methodology. 
Adjenughwure et al. (2015) proposed a hybrid SSAAdaptive Neuro Fuzzy Inference System (ANFIS) for the forecast of monthly passenger demand of two international airports, London Heathrow and Athens Eleftherios Venizelos. The SSA was employed for initial time series decomposition into components, noise elimination, components grouping and time series reconstruction. The hybrid SSA-ANFIS method proved superior to the SSA one, concerning forecasting robustness and accuracy.

Ivanov and Osetrov (2018) proposed a hybrid application of SSA-ANN for the Moscow metro daily traffic forecast. The authors investigated a hybrid SSA-ANN daily passenger traffic forecasting model for Moscow metro. The results revealed a forecasting model with enhanced forecasting efficiency and transport decision management ability.

Lima et al. (2017) tested a model to forecast wind speed from 1 to 24 hours ahead, through SSA and ANN, and proved that it is vigorous and effective for real life applications. Performance model evaluation was realized with the MAPE and MAE statistical criteria. The obtained results demonstrated a robust and reliable wind speed forecasting model.

Siddiquee and Hoque (2017) attempted to forecast road traffic volume at North Bengal corridor of Bangladesh, by using ANN. The main objective of the current paper was daily traffic estimation on selected rural highways of Bangladesh. The authors developed the ANN model with 4 input nodes, one hidden layer with 24 neurons and 2 outputs. Data were segmented into $80 \%-20 \%$ for training and validation and the training algorithm was the back propagation one. The experimental results indicated that the proposed methodology captured initial time series characteristics such as trend or other patterns.

Sun et al. (2018) compared two hybrid methodologies: SSA-Linear Recurrent Formulas (LRF) and SSAANN. In both cases, SSA was employed for decomposition of initial time series into a subset of components, such as trend, seasonal cycles, oscillatory cycles and noise. Decomposed components are fed to LRF and ANN and then the forecasted outputs were compared in terms of statistical criteria such as MAE, RMSE and Coefficient of Determination $\left(R^{2}\right)$. Based on the results, it can be deduced that the hybrid SSA-ANN model accomplished better results than the hybrid SSA-LRF model.

Zubaidi et al. (2018) tried to forecast the monthly water demand by combining SSA with ANN. SSA proved a powerful method used to decompose an initial time series into a subset of components that detected trend and seasonality, and reconstructed a new time series with less noise. Model performance and accuracy of measurements were conducted by using statistical criteria such as RMSE, MAE, Mean Squared Error (MSE) and $R^{2}$.

Chen et al. (2018) developed a hybrid Fuzzy-ANN model for the short-term forecast of the traffic volume of Ho Chi Minh City (Vietnam). The accuracy of the model was measured by using the statistical criteria RMSE, MAPE and $R^{2}$. The proposed model, fed with real traffic data, performed with low values of RMSE and MAPE, and $R^{2}$ values close to 1 , implying a precise model with robust forecasting ability.

\section{The proposed hybrid methodology}

Making use of artificial intelligence algorithms for the analysis and forecasting of traffic volume is an innovative application of the relevant knowledge in the field of transportation, which presents challenges and variety, depending on the differentiation of qualitative and quantitative characteristics that describe the time series (Karlaftis and Vlahogianni, 2011; Štencl and Lendel, 2012). The architecture of the proposed hybrid SSA-ANN methodology, (Fig. 1), is a modern approach for the modeling of traffic volume forecasting.

The proposed hybrid methodology for traffic volume forecasting is based on the combination of SSA and the use of conventional ANN. The aim of the methodology is to optimize the forecasting ability according to the following steps (Kolidakis and Botzoris, 2018):

- decomposition, by using the SSA, of the initial time series,

- elimination of the noise component and (optionally) grouping of components with common characteristics,

- response forecasting, by using conventional ANN, of each main component of the initial time series,

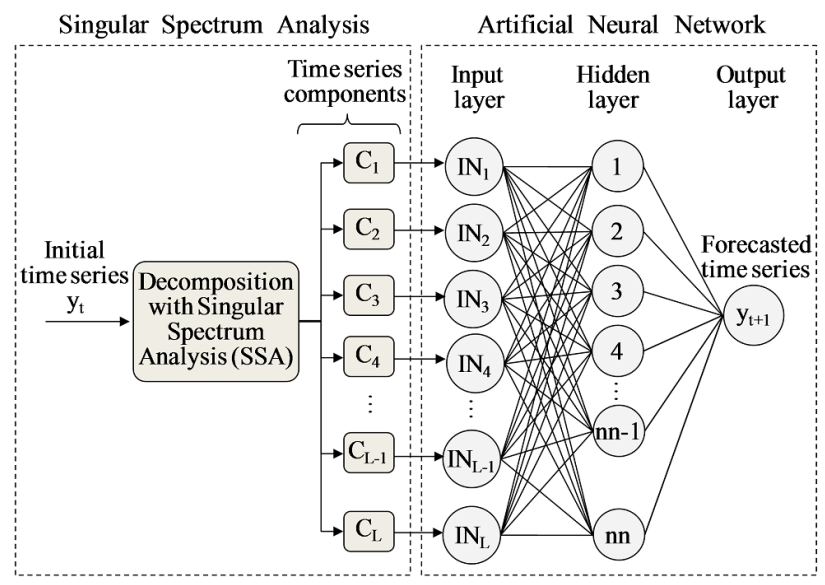

Fig. 1 Architecture of the proposed hybrid SSA-ANN methodology 
- reconstruction of the forecasting response of each main component,

- enhanced results from hybrid model comparison.

- comparison of results of hybrid SSA-ANN and conventional ANN, and ex post forecasting ability assessment.

SSA methodology is quite effective for time series analysis and is commonly applied to wide research areas. The most prominent quality of SSA is to disassemble initial time series into component time series that can each be discerned as a trend component, a seasonal or periodical component and noise component.

SSA methodology comprises two stages and four steps: the decomposition stage which is analyzed to embedding and singular value decomposition; and the reconstruction stage which is sub-divided into grouping and diagonal averaging (Golyandina and Zhigljavsky, 2013).

Embedding projects the initial time series into a sequence of lagged vectors of size $L$ by forming $K=T-L+1$ lagged vectors $X_{i}=\left\{x_{i}, x_{i+1}, \ldots, x_{i+L-1}\right\}^{T}, i=1, \ldots, K$. The way those are calculated is reflected by Eq. (1):

$$
X=\left[X_{1}, X_{2}, \ldots, X_{K}\right]=\left(x_{i j}\right)_{i, j=1}^{L, K} \text {. }
$$

The above matrix is called trajectory matrix and it is a Henkel matrix since $X$ has identical data elements on anti-diagonals (Golyandina and Zhigljavsky, 2013).

From the matrix $X$, we describe a $X X^{\mathrm{T}}$ covariance matrix. Singular value decomposition $(S V D)$ of $X X^{\mathrm{T}}$ delivers a set of $L$ eigenvalues in decreasing order of magnitude and the corresponding eigenvectors $U_{1}, U_{2}, \ldots, U_{L}$. Then, the SVD of the trajectory matrix can be written as $X=\left[X_{1}, X_{2}, \ldots, X_{L}\right]$, where $X_{i}=\sqrt{\lambda_{i}} U_{i} V_{i}^{T}$.

The Grouping partitions the set of indices $\{1,2, \ldots, L\}$ into $r$ disjoints subsets. Let $I=\{i 1, i 2, \ldots, i r\}$ be a group of $r$ selected eigentriples, and $X_{I}=X_{i 1}, X_{i 2}, \ldots, X_{i r}$, where $X_{I}$ are the related to the signal eigentriples, while the remaining $(L-r)$ eigentriples denote the time series related to the error.

Diagonal averaging is the final step to reconstruct the components of a time series. The procedure is finalized with transformation of each of the terms $X_{I}=X_{i 1}, X_{i 2}, \ldots, X_{i r}$ into reconstructed time series $F_{i 1}, F_{i 2}, \ldots, F_{i r}$ via the Hankelization process $H($ ) or diagonal averaging: assuming $b_{i j}$ as an element of a generic matrix $B$, the $s^{\text {th }}$ term of the reconstructed time series could be obtained by $b_{i j}$, if $i+j=s+1$. When the final step is accomplished, the reconstructed time series approximates the initial time series, while eliminating noise components.

\section{Implementation and results}

\subsection{Case study}

In order to verify the effectiveness and reliability of the proposed hybrid methodology, an application is developed, by using hourly traffic data of passenger vehicles from the toll station of Iasmos along Egnatia Motorway, Northern Greece, (Fig. 2). Hourly time interval for data recording begins on January 01, 2018 and ends on July 31, 2018, which means that there are available 5088 hourly traffic volume records (Fig. 3).

\subsection{Evaluation criteria}

The most common statistical evaluation criteria are the Mean Absolute Error (MAE), the Mean Squared Error (MSE), and the Root Mean Squared Error (RMSE). The MAE is calculated from Eq. (2), the MSE is calculated from Eq. (3), whereas the RMSE is calculated from Eq. (4) (Profillidis and Botzoris, 2018):

$$
\begin{aligned}
& \text { MAE }=\frac{1}{n} \sum_{i=1}^{n}\left|y_{i}-\tilde{y}_{i}\right|, \\
& \text { MSE }=\frac{1}{n} \sum_{i=1}^{n}\left(y_{i}-\tilde{y}_{i}\right)^{2}, \\
& \text { RMSE }=\sqrt{\frac{1}{n} \sum_{i=1}^{n}\left(y_{i}-\tilde{y}_{i}\right)^{2},}
\end{aligned}
$$

where $y_{i}$ are the actual values of the initial time series, $\tilde{y}_{i}$ the forecasted by the model values, and $n$ the number of the time series available data.

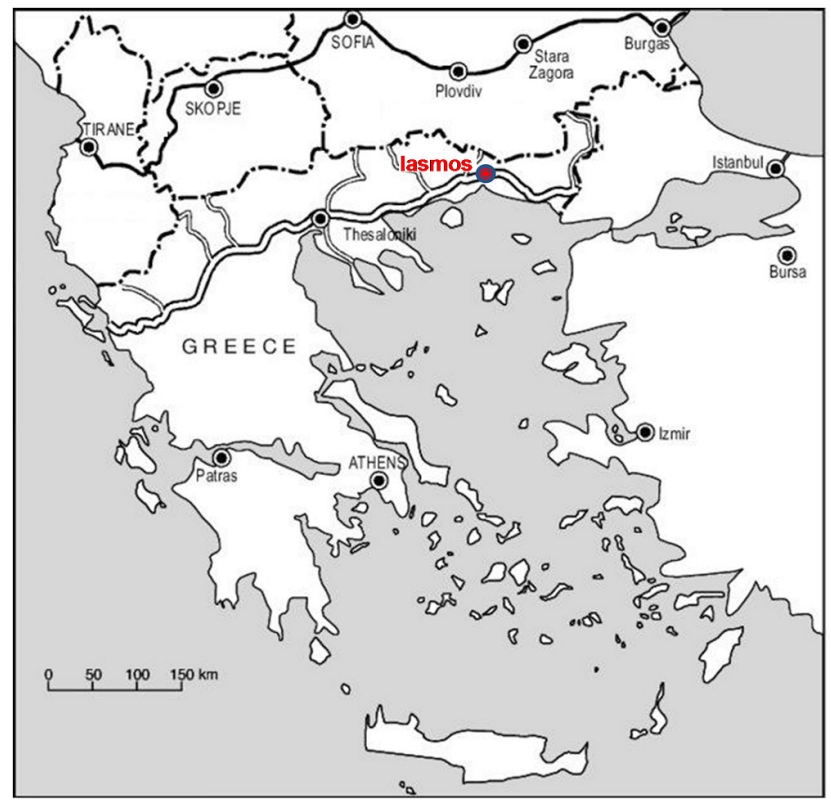

Fig. 2 The toll station of Iasmos along Egnatia Motorway (Polyzos, 2009) 


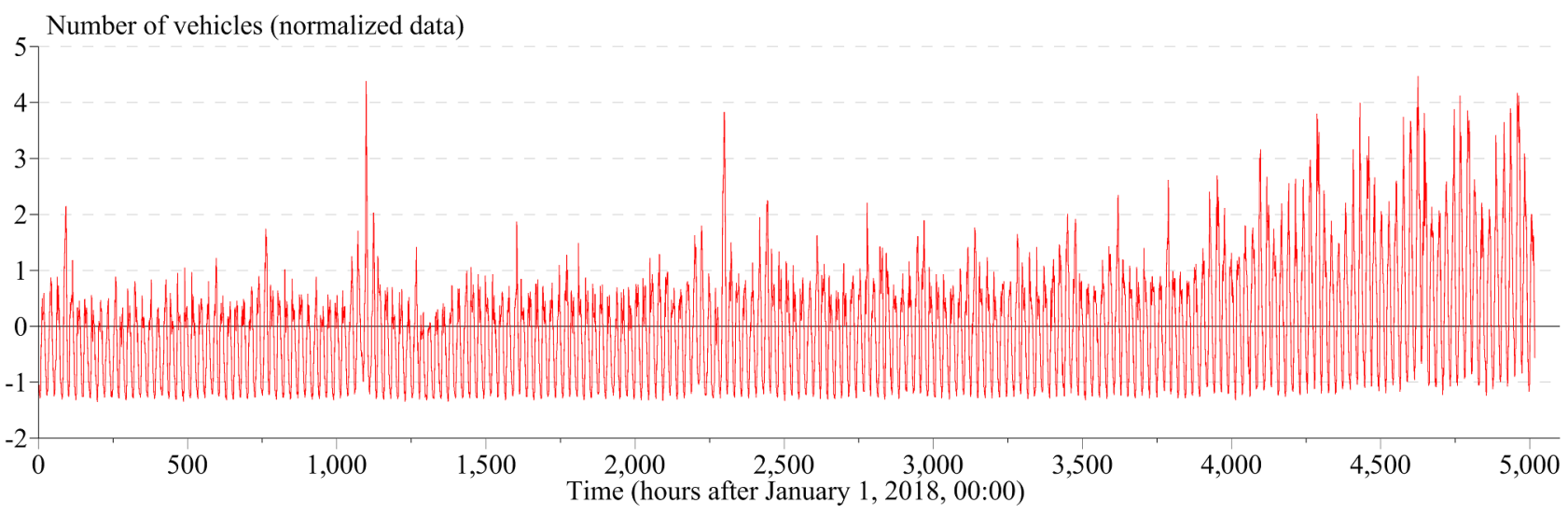

Fig. 3 Iasmos toll station hourly traffic volume

In addition to MAE, MSE, and RMSE, Theil's $U$ can be used as a dimensionless criterion for the comparative evaluation of the forecasting ability of various models. Theil's $U$ is calculated from Eq. (5):

$$
U=\frac{\sqrt{\frac{1}{n} \sum_{i=1}^{n}\left(y_{i}-\tilde{y}_{i}\right)^{2}}}{\sqrt{\frac{1}{n} \sum_{i=1}^{n}\left(y_{i}\right)^{2}}+\sqrt{\frac{1}{n} \sum_{i=1}^{n}\left(\tilde{y}_{i}\right)^{2}}},(i=1,2, \ldots, n) .
$$

If the forecasting model's $U$ is estimated to be equal to 0 , then the forecasting ability for that model is perfect, when the forecasting model's $U$ is estimated to be equal to 1 , the forecasting model hasn't any forecasting ability. Practically, values of $U$ less than 0.50 are acceptable whereas values of $U$ less than 0.10 are excellent.

Theil's $U$ is further analyzed with regard to three proportions, the bias $U^{M}$ (indicates the systematic error of a model and the preferred values are close to 0 ), the variance $U^{S}$, and the covariance $U^{C}$ (indicates the avoidable random error of a model), which can be calculated with the help of the Eq. (6), Eq. (7), Eq. (8) and Eq. (9) (Profillidis and Botzoris, 2018):

$$
\begin{aligned}
& U^{M}=\frac{\left(\overline{y_{i}}-\overline{\tilde{y}_{i}}\right)^{2}}{\frac{1}{n} \sum_{i=1}^{n}\left(y_{i}-\tilde{y}_{i}\right)^{2}}, \\
& U^{S}=\frac{\left(\sigma_{y}-\sigma_{\tilde{y}}\right)^{2}}{\frac{1}{n} \sum_{i=1}^{n}\left(y_{i}-\tilde{y}_{i}\right)^{2}}, \\
& U^{C}=\frac{2 *\left(1-r_{y \tilde{y}}\right) \sigma_{y} \sigma_{\tilde{y}}}{\frac{1}{n} \sum_{i=1}^{n}\left(y_{i}-\tilde{y}_{i}\right)^{2}}, \\
& U^{M}+U^{S}+U^{C}=1,
\end{aligned}
$$

where

$\sigma_{y} \quad$ actual values' $\left(\sigma_{i}\right)$ standard deviation.

$\sigma_{\tilde{y}} \quad$ forecasted values' $\left(\tilde{y}_{i}\right)$ standard deviation.

$r_{y \tilde{y}} \quad$ the coefficient of correlation between the actual $\left(y_{i}\right)$ and the forecasted values $\left(\tilde{y}_{i}\right)$.

\subsection{Ex post evaluation}

The validity and the consistency of a calibrated model can be checked by using various methods and statistical criteria. An easy method to use is the comparison of the out-of-sample forecasts of a model with the actual values that were recorded after the model training and testing (Profillidis and Botzoris, 2006). Thus, we can compare provisional forecasts of the model with recorded values and, thus, assess the reliability of the model.

\subsection{Application and results}

We suppose that traffic volume for the next hour is a function of the traffic volume of the previous $\operatorname{Lag}$ hours ( $\operatorname{Lag}=1,2$, $\ldots$ and $L a g \leq 5088$ ). Therefore, the conventional ANN architecture is described by Eq. (10):

$y_{t+1}=f\left(y_{t}, y_{t-1}, y_{t-2}, \ldots, y_{t-\text { Lag }}\right)+u$,

where

$y_{t+1} \quad$ the measured (or forecasted) value of traffic volume of the next hour.

$y_{t} \quad$ the measured traffic volume for the present hour. $y_{t-1} \quad$ the measured traffic volume for the previous hour. Lag $\quad 1,2, \ldots, 5088$.

$u \quad$ the error term.

Window length selection is a critical parameter that describes the number of past values that influence the future output. This factor is significant for the architecture of the suggested forecasting model but unfortunately 
there is no rule for an accurate definition, but only empirical approaches or rule of thumbs. In our case study, since our values refer to hourly measurements, the selection window length at 72 values (past 3 days hourly values) is based on empirical expertise and intuitive approach from previous research (Kolidakis et al., 2018). In the same approach, we select the number of neurons range to be 20 , since we have simulated in previous research a plethora of combinations for different number of neurons (Kolidakis et al., 2018). Therefore, we select ANN topology with one hidden layer, $L a g=72$ and number of neurons (nn) 20 .

As aforementioned, only two elements are prerequired in SSA: window length $L a g$ and number of components $r$. Lag is defined above at 72 and in Fig. 4 principal components (30 components) are extracted by the decomposition of the initial time series by using SSA (normalized values). Also, the number of components $r$ is based on the theory of separability, which describes how well the components can be separated. A standard measure based on the involvement of each element to the variance of initial time series, is evaluated from Eq. (11) as:

$$
\frac{\lambda_{i}}{\sum_{i=1}^{d} \lambda_{i}}
$$

where

$\lambda_{i} \quad$ is the eigenvalue $i$ to the corresponding eigenvector $i$.

d $\quad 1,2, \ldots$, Lag.

In general, we select $r$ out of Lag components, so that the summation of their contribution is above a crucial predetermined value (e.g., greater than $90 \%$ or $95 \%$ ). Usually, eigenvalues with low contribution are considered as noise (Briceño et al., 2013).

A valuable insight is possible by evaluating breaking points in eigenvalue spectrum (Fig. 5). Noise time series provides a slowly decreasing series of singular values (Hassani and Thomakos, 2010). In our case, a major drop in values happens around the component 30 , which can be assumed as the starting point of noise components.

Figs. 6 and 7 compare the actual hourly traffic volume at Iasmos toll station with the forecasted traffic volume by the proposed conventional ANN and the hybrid SSA-ANN methodology. This graphical representation emphasizes a significant overlap between the actual and the forecasted values by the conventional ANN and the hybrid SSA-ANN, implying that we successfully forecast the hourly traffic volume. Moreover, from Figs. 8 and 9, we benchmark ex post forecast of the proposed hybrid SSA-ANN upon conventional ANN and identify visually the forecasting superiority of the proposed hybrid implementation.

Table 1 provides statistical criteria comparison of hybrid SSA-ANN versus conventional ANN. All critical criteria are improved in the case of hybrid model $\left(R^{2}\right.$ is improved by $8.30 \%$, RMSE is improved by $44.45 \%$ and MAE is improved by $40.28 \%$ ). Theil's $U$ is less than 0.5 , which is satisfactory, whereas in the case of hybrid model is close to 0.1 (0.1293), which is considered as excellent. $U^{M}$ remains close to zero, which is a very good indication for the systemic error.

In the case of ex post forecasting, Table 2 provides the comparison of hybrid SSA-ANN versus conventional ANN. In addition, Theil's $U$ remains less than 0.5 and $U^{M}$ close to zero and all critical criteria are improved in the case of the hybrid model ( $R^{2}$ is improved by $6.06 \%$, RMSE is improved by $11.31 \%$ and MAE is improved by $8.76 \%$ ).

\section{Conclusions and further research}

Accurate traffic volume forecasts are vital for decision makers, transportation engineers, local and national authorities. Robust forecasts are critical for the decision-making with regard to long-term and short-term planning. Traffic volume forecasting is important in long-range planning, providing the decision makers with knowledge for transportation systems maintenance and expanding.

The paper presents a comparative evaluation of hybrid SSA and ANN against conventional ANN, applied on real time intraday traffic volume forecasting. The main research objective was to assess the applicability of intraday traffic volume forecasting, based on toll station measurements for real data ex post forecasting. The proposed methodology was implemented and evaluated upon a custom developed forecasting software toolbox, based on the software Mathworks MatLab, by using real data from the Iasmos toll station, along Egnatia Motorway (Northern Greece).

Experimental results demonstrated a superior ex post forecasting accuracy of the proposed hybrid forecasting methodology against conventional ANN, when compared to performance of typical statistical criteria. These results clarified that the hybrid model could improve the forecasting accuracy of a conventional ANN model in intraday traffic volume forecasting, certified by ex post forecasting assessment.

In recent years, traffic volume forecasting literature has achieved major advances. Embedding hybrid forecasting algorithm in an Intelligent Transport System could provide an advanced decision support tool for transportation 

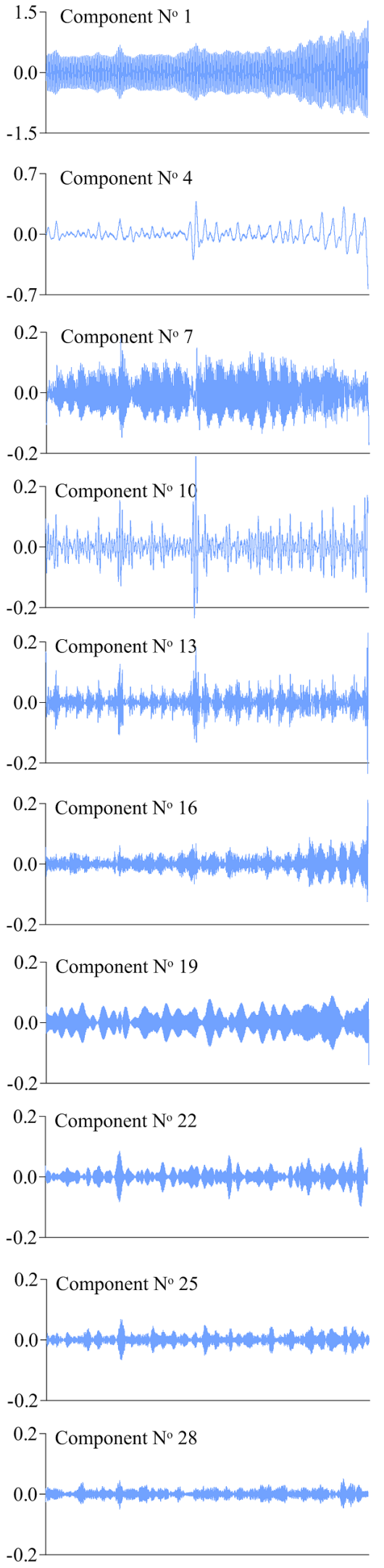
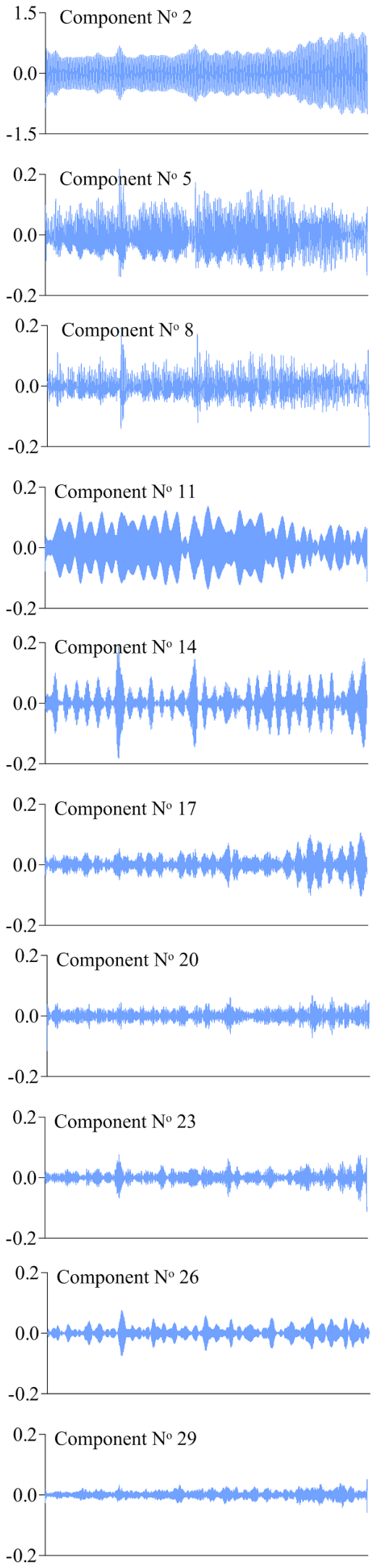
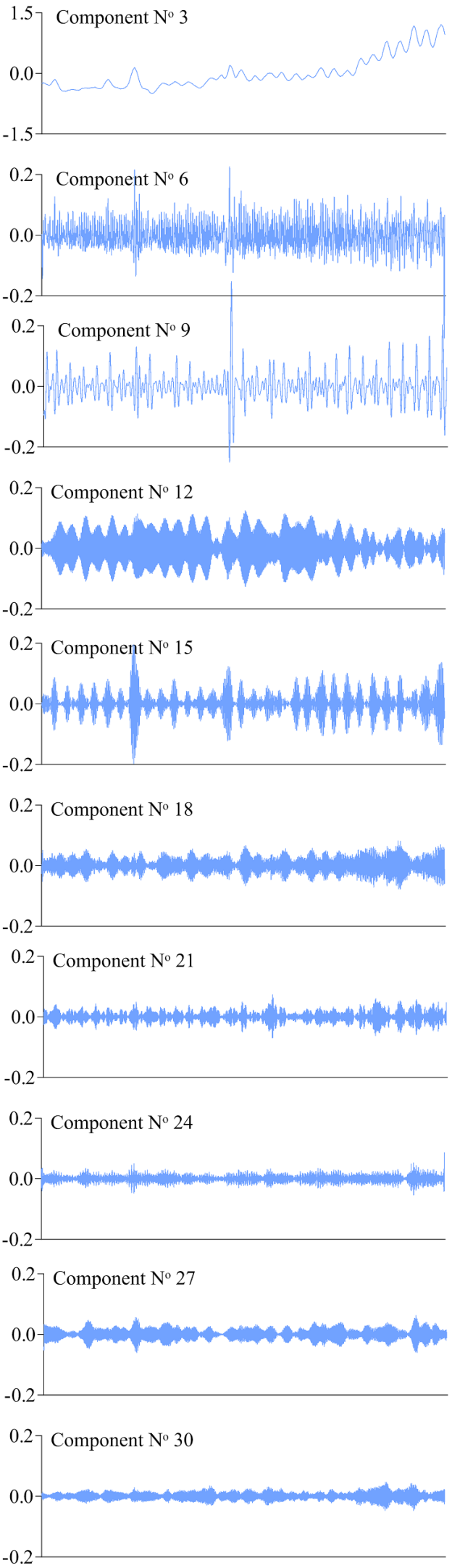

Fig. 4 Principal components extracted by decomposition of initial time series using SSA (normalized values) 


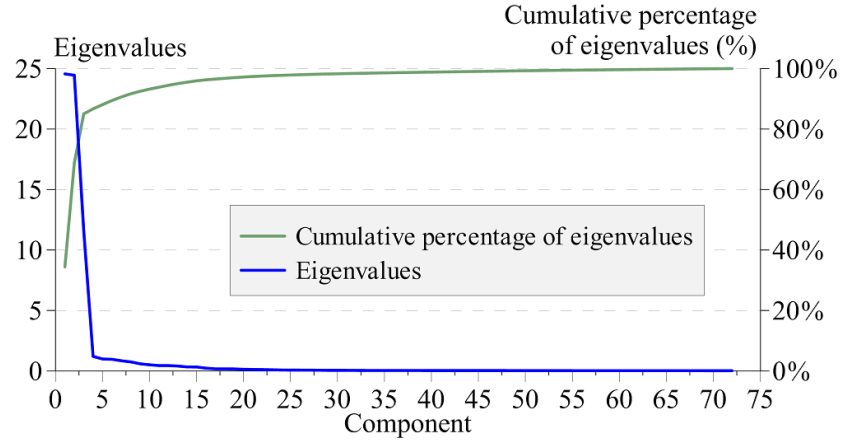

Fig. 5 Eigenvalues and cumulative percentage of eigenvalues (\%) system maintenance, operation and management, while it could enable a proactive decision to relieve environmental and economic impacts of extended transportation systems congestion.

Identified reasons that testify the robustness of the hybrid SSA-ANN methodology can be derived from the outcomes of time series SSA decomposition. Analyzing the initial time series into components, using SSA, results in simpler components that are easier to exploit and forecast trends, seasonality and other main frequency components.

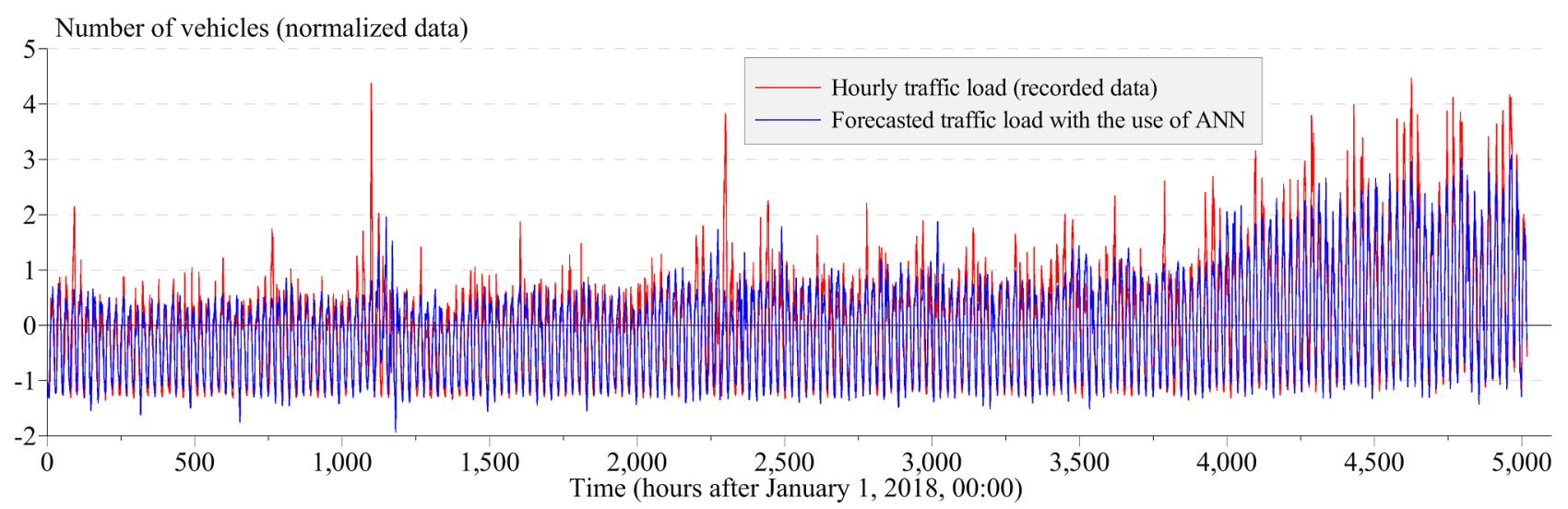

Fig. 6 Comparison of the forecasted, using conventional ANN, with the original time series

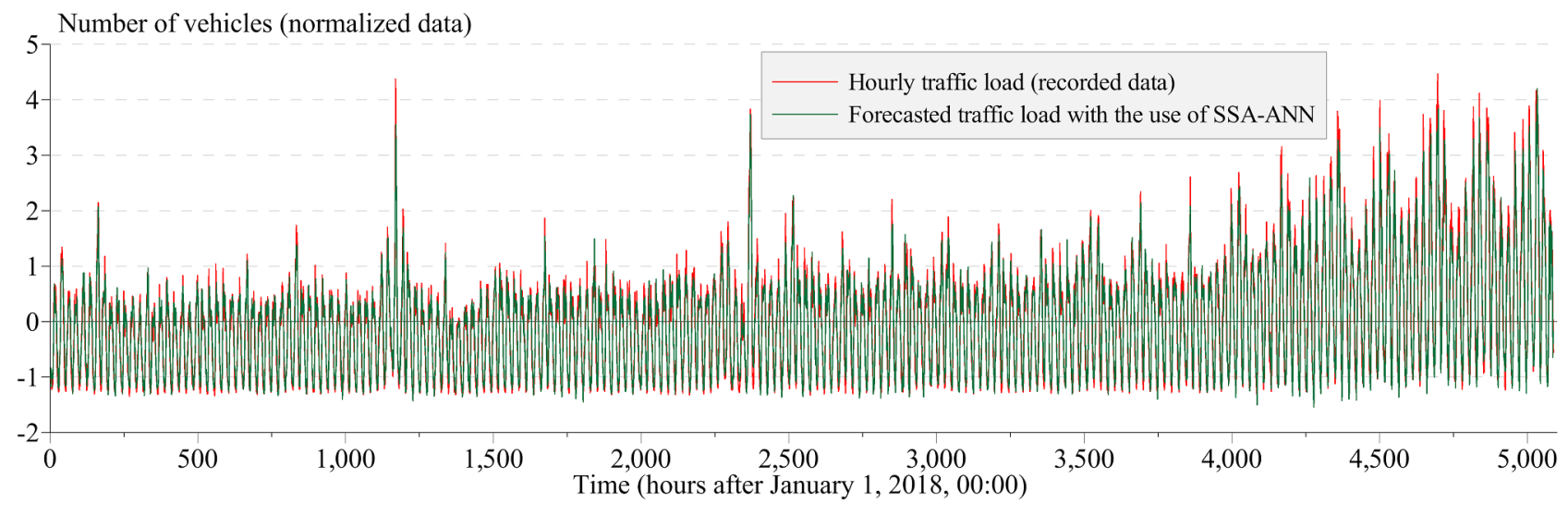

Fig. 7 Comparison of the forecasted, using the hybrid SSA-ANN method, with the original time series

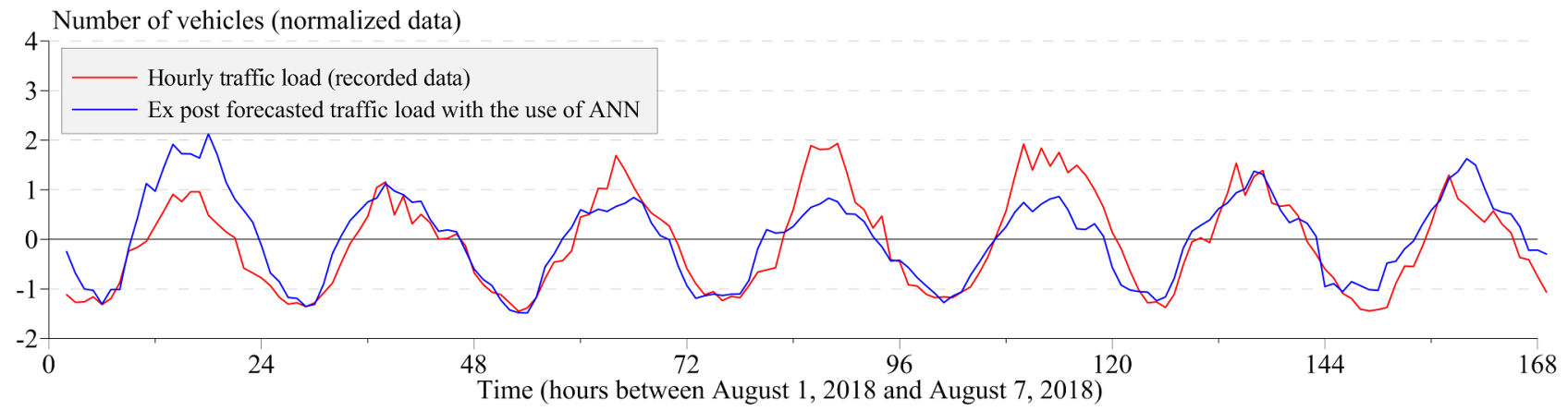

Fig. 8 Ex post forecast assessment - comparison of the forecasted, using conventional ANN, with the original time series 


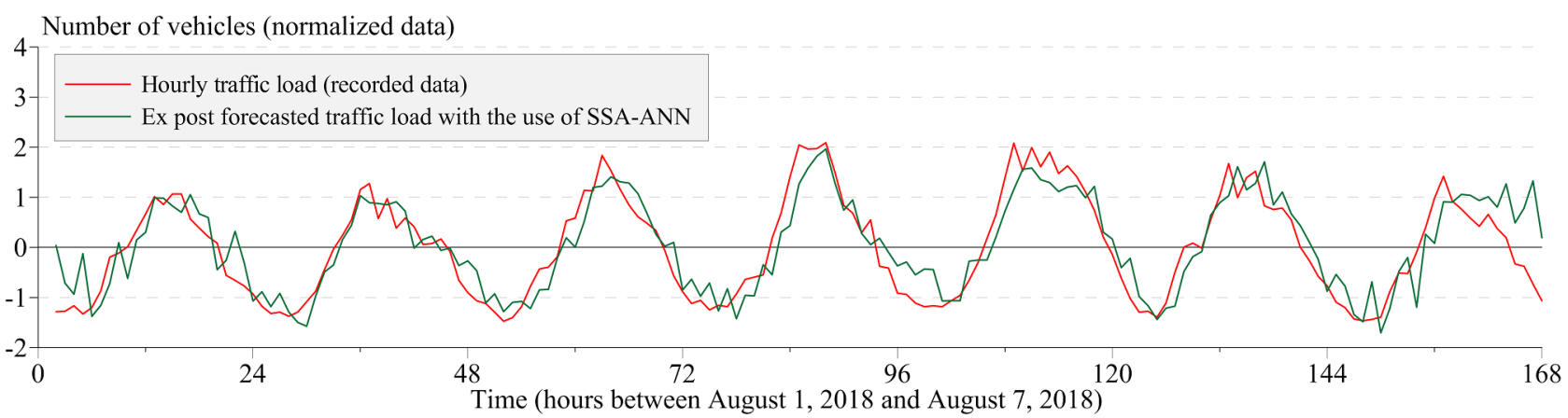

Fig. 9 Ex post forecast assessment - comparison of the forecasted, using the hybrid SSA-ANN method, with the original time series

Table 1 Evaluation, by using statistical criteria, of the results of the proposed hybrid SSA-ANN methodology

\begin{tabular}{lccc}
\hline Criteria & $\begin{array}{c}\text { Conventional } \\
\text { ANN }\end{array}$ & $\begin{array}{c}\text { Hybrid } \\
\text { SSA-ANN }\end{array}$ & \% improvement \\
\hline$R^{2}$ & 0.8960 & 0.9704 & $8.30 \%$ \\
RMSE & 0.4461 & 0.2478 & $-44.45 \%$ \\
MAE & 0.3116 & 0.1861 & $-40.28 \%$ \\
Theil's $U$ & 0.2387 & 0.1293 & $-45.83 \%$ \\
$U^{M}$ & 0.0031 & 0.0023 & $-25.81 \%$ \\
$U^{C}$ & 0.9074 & 0.8837 & $-2.61 \%$ \\
$U^{S}$ & 0.0895 & 0.1140 & $27.37 \%$ \\
\hline
\end{tabular}

Table 2 Evaluation, by using statistical criteria, of the results of the proposed hybrid SSA-ANN methodology for ex post forecasting assessment

\begin{tabular}{lccc}
\hline Criteria & $\begin{array}{c}\text { Conventional } \\
\text { ANN }\end{array}$ & $\begin{array}{c}\text { Hybrid } \\
\text { SSA-ANN }\end{array}$ & \% improvement \\
\hline$R^{2}$ & 0.8287 & 0.8789 & $6.06 \%$ \\
RMSE & 0.5403 & 0.4792 & $-11.31 \%$ \\
MAE & 0.4076 & 0.3719 & $-8.76 \%$ \\
Theil's $U$ & 0.3014 & 0.2545 & $-15.56 \%$ \\
$U^{M}$ & 0.0101 & 0.0023 & $-77.23 \%$ \\
$U^{C}$ & 0.8025 & 0.9595 & $19.56 \%$ \\
$U^{S}$ & 0.1874 & 0.0382 & $-79.62 \%$ \\
\hline
\end{tabular}

\section{References}

Adjenughwure, K., Balopoulos, V., Botzoris, G. (2015) "Singular Spectrum Analysis - Hybrid Forecasting Methods with Application to Air Transport Demand", presented at $7^{\text {th }}$ International Congress on Transport Research, Thessaloniki, Greece, Nov. 5-6, 2015. [online] Available at: http://ictr.imet.gr/ Portals/0/Documents/7thCongress/Papers/ICTR_2015_submission_33.pdf [Accessed: 31 January 2019]

Aleksandrov, T. (2009) "A Method of Trend Extraction Using Singular Spectrum Analysis", REVSTAT-Statistical Journal, 7(1), pp. 1-22. [online] Available at: https://arxiv.org/pdf/0804.3367.pdf [Accessed: 31 January 2019]
Moreover, the above-mentioned simplicity performs noise removal components, with minor signal contribution.

Traffic volume forecasting is a very stimulating engineering task. Traffic volume time series have a stochastic character and can be affected by various factors. Therefore, more effort should be placed towards the goal of increasing the forecasting accuracy. Future research can be focused upon the following sectors:

- Preprocessing data methodologies in order to diminish processing time and forecasting errors.

- Develop new forecasting models upon the idea of hybrid or combined methodologies.

- Include clustering toolboxes that would extract transportation systems users' profiles and involve them in forecasting methodology.

The proposed hybrid methodology can be applied to the national motorway system at the specific and other countries, in order to identify similarities and differences of the transport system characteristics that can improve the forecasting ability and accuracy.

\section{Acknowledgement}

The authors would like to express their gratitude to Egnatia Odos S. A. for the concession of the raw data.

Andersson, M., Brundell-Freij, K., Eliasson, J. (2017)"Validation of aggregate reference forecasts for passenger transport", Transportation Research Part A: Policy and Practice, 96, pp. 101-118. https://doi.org/10.1016/j.tra.2016.12.008

Blinova, T. O. (2007) "Analysis of Possibility of Using Neural Network to Forecast Passenger Traffic Flows in Russia", Aviation, 11(1), pp. 28-34.

https://doi.org/10.3846/16487788.2007.9635952

Box, G. E. P., Jenkins, G. M., Reinsel, G. C., Ljung, G. M. (2015) "Time Series Analysis: Forecasting and Control", 5th ed., John Wiley \& Sons, Hoboken, NJ, USA. 
Briceño, H., Rocco, C. M., Zio, E. (2013) "Singular Spectrum Analysis for Forecasting Electric Load Demand", Chemical Engineering Transactions, 33, pp. 919-924.

https://doi.org/10.3303/CET1333154

Chen, J.-F., Lo, S.-K., Do, Q. H. (2018) "Forecasting Short-Term Traffic Flow by Fuzzy Wavelet Neural Network with Parameters Optimized by Biogeography-Based Optimization Algorithm", Computational Intelligence and Neuroscience, 2018, Article ID: 5469428. https://doi.org/10.1155/2018/5469428

Golyandina, N., Zhigljavsky, A. (2013) "Singular Spectrum Analysis for Time Series", 1st ed., Springer, Berlin, Germany. https://doi.org/10.1007/978-3-642-34913-3

Hassani, H. (2007) "Singular Spectrum Analysis: Methodology and Comparison", Journal of Data Science, 5(2), pp. 239-257. [online] Available at: https://mpra.ub.uni-muenchen.de/4991/ [Accessed: 31 January 2019]

Hassani, H., Thomakos, D. (2010) "A review on singular spectrum analysis for economic and finance time series", Statistics and Its Interface, 3(3), pp. 377-397.

https://doi.org/10.4310/SII.2010.v3.n3.a11

Ivanov, V., Osetrov, E. (2018) "Application of Artificial Neural Networks and Singular-Spectral Analysis in Forecasting the Daily Traffic in the Moscow Metro", In: Nineth International Conference on Mathematical Modeling and Computational Physics (MMCP 2017), Dubna, Russia, article ID: 05009. https://doi.org/10.1051/epjconf/201817305009

Karlaftis, M. G., Vlahogianni, E. I. (2011) "Statistical methods versus neural networks in transportation research: Differences, similarities and some insights", Transportation Research Part C: Emerging Technologies, 19(3), pp. 387-399. https://doi.org/10.1016/j.trc.2010.10.004

Kolidakis, S. Z., Botzoris, G. N. (2018) "Enhanced Air Traffic Demand Forecasting Using Artificial Intelligence", In: 6 $^{\text {th }}$ QUAESTI Virtual Multidisciplinary Conference, Zilina, Slovakia, pp. 126-131. https://doi.org/10.18638/quaesti.2018.6.1.383

Kolidakis, S., Botzoris, G., Profillidis, V., Lemonakis, P. (2018) "Real-time road traffic forecasts - a hybrid approach using artificial intelligence and Singular Spectrum Analysis", In: $5^{\text {th }}$ Conference Economics of Natural Resources and the Environment, Volos, Greece, pp. 165-174. [online] Available at: http://envecon.econ.uth. gr/5th_Conference_Proceedings.pdf [Accessed: 31 January 2019]

Lima, C., de Oliveira Castellani, V. L., Pessanha, J. F. M., Soares, A. (2017) "A Model to Forecast Wind Speed Through Singular Spectrum Analysis and Artificial Neural Networks", In: International Conference on Artificial Intelligence, Las Vegas, NV, USA, pp. 235-240. [online] Available at: https://csce.ucmss. com/cr/books/2017/LFS/CSREA2017/ICA6119.pdf [Accessed: 31 January 2019]

Pamuła, T. (2012) "Traffic Flow Analysis Based on the Real Data Using Neural Networks", In: $12^{\text {th }}$ International Conference on Transport Systems Telematics, Katowice-Ustroń, Poland, pp. 364-371. https://doi.org/10.1007/978-3-642-34050-5_41

Pamuła, T. (2013) "Short-Term Traffic Flow Forecasting Method Based on the Data from Video Detectors Using a Neural Network", In: $13^{\text {th }}$ International Conference on Transport Systems Telematics, Katowice-Ustroń, Poland, pp. 147-154. https://doi.org/10.1007/978-3-642-41647-7_19
Polyzos, S. (2009) "The Egnatia Motorway and the Changes in Interregional Trade in Greece: An Ex Ante Assessment", European Spatial Research and Policy, 16(2), pp. 23-47. https://doi.org/10.2478/v10105-009-0011-7

Profillidis, V. A., Botzoris, G. N. (2006) "Econometric models for the forecast of passenger demand in Greece", Journal of Statistics and Management Systems, 9(1), pp. 37-54.

https://doi.org/10.1080/09720510.2006.10701192

Profillidis, V., Botzoris, G. (2018) "Modeling of Transport Demand: Analyzing, Calculating, and Forecasting Transport Demand", 1st ed., Elsevier, Oxford, UK. https://doi.org/10.1016/C2016-0-00793-3

Schimbinschi, F., Nguyen, X. V., Bailey, J., Leckie, C., Vu, H., Kotagiri, R. (2015) "Traffic forecasting in complex urban networks: Leveraging big data and machine learning", In: IEEE International Conference on Big Data, Santa Clara, CA, USA, pp. 1019-1024. https://doi.org/10.1109/BigData.2015.7363854

Siddiquee, M. S. A., Hoque, S. (2017) "Predicting the Daily Traffic Volume from Hourly Traffic Data Using Artificial Neural Network", Neural Network World, 27(3), pp. 283-294. https://doi.org/10.14311/NNW.2017.27.015

Štencl, M., Lendel, V. (2012) "Application of selected artificial intelligence methods in terms of transport and intelligent transport systems", Periodica Polytechnica Transportation Engineering, 40(1), pp. 11-16. https://doi.org/10.3311/pp.tr.2012-1.02

Sun, M., Li, X., Kim, G. (2018) "Precipitation analysis and forecasting using singular spectrum analysis with artificial neural networks", Cluster Computing, 21, pp. 1-8. https://doi.org/10.1007/s10586-018-1713-2

Teodorović, D., Janić, M. (2017) "Transportation Engineering: Theory, Practice and Modeling", Butterworth-Heinemann, Oxford, UK. https://doi.org/10.1016/C2015-0-00596-2

Vlahogianni, E. I., Karlaftis, M. G., Golias, J. C. (2008) "Temporal Evolution of Short-Term Urban Traffic Flow: A Nonlinear Dynamics Approach", Computer-Aided Civil and Infrastructure Engineering, 23(7), pp. 536-548. https://doi.org/10.1111/j.1467-8667.2008.00554.x

Vlahogianni, E. I., Karlaftis, M. G., Golias, J. C. (2014) "Short-term traffic forecasting: Where we are and where we're going", Transportation Research Part C: Emerging Technologies, 43(1), pp. 3-19. https://doi.org/10.1016/j.trc.2014.01.005

Wu, J., Zhong, L., Li, L., Lu, A. (2013) "A Prediction Model Based on Time Series Data in Intelligent Transportation System", In: $4^{\text {th }}$ International Conference on Information Computing and Applications, Singapore, Singapore, pp. 420-429. https:/doi.org/10.1007/978-3-642-53703-5_43

Zhang, G. P, Qi, M. (2005) "Neural network forecasting for seasonal and trend time series", European Journal of Operational Research, 160(2), pp. 501-514. https://doi.org/10.1016/j.ejor.2003.08.037

Zubaidi, S. L., Dooley, J., Alkhaddar, R. M., Abdellatif, M., Al-Bugharbee, H., Ortega-Martorell, S. (2018) "A Novel approach for predicting monthly water demand by combining singular spectrum analysis with neural networks", Journal of Hydrology, 561, pp. 136-145.

https://doi.org/10.1016/j.jhydrol.2018.03.047 